\title{
Efeito de um programa de reeducação alimentar na capacidade funcional de mulheres
}

\author{
Effect of a food reeducation program on the functional capacity of women
}

\author{
Carlos Leandro Tiggemanna, Indianara Cristina Gonçalves ${ }^{b}$, Helena Conrad dos Santosc, \\ Marco Aurélio Weber ${ }^{d}$, Jéssica Luana Dornelles da Costae, Caroline Pieta Dias ${ }^{f}$ \\ a Educador Físico. Doutor em Ciência do Movimento Humano. Universidade do Vale do Taquari (Univates). \\ ${ }^{\mathrm{b}}$ Educador Físico graduado pela Univates. \\ c Nutricionista graduada pela Univates. \\ dAdministrador. Cooperativa Certel. \\ educador Físico graduado pela Univates. \\ ¿Educador Física. Doutora em Ciência do Movimento Humano. Universidade Federal do Rio Grande do Sul.
}

Introdução: A obesidade é um problema de saúde pública que compromete vários parâmetros da saúde dos sujeitos, entre eles a capacidade funcional (CF).

Objetivo: Avaliar o efeito da participação de mulheres em um programa de reeducação alimentar na capacidade funcional.

Materiais e Métodos: Estudo de característica longitudinal retrospectiva, contou com uma amostra de 794 mulheres (48,4 $\pm 12,3$ anos; índice de massa corporal de 30,8 $\left.\pm 5,2 \mathrm{~kg} / \mathrm{m}^{2}-\mathrm{IMC}\right)$. Os sujeitos participaram semanalmente de um programa de reeducação alimentar durante 20 semanas. A massa corporal (MC) e a prática de exercícios físicos (autorrelato) foram mensuradas semanalmente, sendo a CF (Health Assessment Questionnaire - HAQ20; escores de 0 a 3) e o perímetro abdominal avaliado ao início e final do programa. A amostra total foi dividida em subgrupos quanto ao IMC, magnitude de redução de $\mathrm{MC}$, prática de exercícios físicos e idade. As variáveis foram comparadas por meio do teste de Wilcoxon (pré e pós) e o teste Mann-Whitney (entre os subgrupos; $p \leq 0,05$ ).

Resultados: Melhora significativa da CF entre os participantes $(0,17 \pm 0,31$ vs $0,07 \pm 0,21 ; p<0,001)$, assim como, nos parâmetros antropométricos (MC 80,66 14,58 vs 75,48 $\pm 14,03 ; p<0,001)$. Todos os subgrupos apresentaram melhora da CF no transcorrer do tempo, sendo que somente os subgrupos IMC e idade apresentaram diferenças significativas da CF entre si.

Conclusão: Conclui-se que a participação de mulheres em um programa de reeducação alimentar promove uma melhora da sua $\mathrm{CF}$, reduzindo vários parâmetros da composição corporal, sendo que somente a idade e o IMC apresentam influência na CF.

Palavras-chave: obesidade; índice de massa corporal; mulheres; pessoas com deficiência; perda de peso.

Introduction: Obesity is a public health problem that compromises several health parameters of the subjects, including functional capacity (FC).

Objective: To evaluate the effect of the participation of women in a food reeducation program on FC.

Materials and Methods: This is a longitudinal retrospective study including a sample of 794 women (48.4 \pm 12.3 years; body mass index of $30.8 \pm 5.2 \mathrm{~kg} / \mathrm{m}^{2}-\mathrm{BMI}$ ). Subjects participated weekly in a food reeducation program for 20 weeks. Body mass (BM) and physical exercise (self-report) were measured weekly, with the Health Assessment Questionnaire (HRQ20) and the abdominal perimeter evaluated at the beginning and at the end of the program. The total sample was divided into subgroups for BMI, magnitude of BM reduction, physical exercise practice and age. The variables were compared using the Wilcoxon test (pre and post) and the Mann-Whitney test (among the subgroups, $p \leq 0.05$ ).

Results: Significant improvement of the FC among participants $(0.17 \pm 0.31 \mathrm{vs} 0.07 \pm 0.21 ; p<0.001)$, as well as in the anthropometric parameters (BM 80.66 \pm 14.58 vs 75.48 $\pm 14.03 ; p<0.001$ ). All subgroups showed improvement of the FC over time, and only the $\mathrm{BMI}$ and age subgroups presented significant differences between the two groups.

Conclusion: It is concluded that the participation of women in a program of dietary reeducation promotes an improvement of their $F C$, reducing several parameters of the body composition, in a way that only age and BMI influenced FC.

Keywords: obesity; body mass index; women; disable persons; weight loss.

\section{Correspondência:}

JÉSSICA LUANA DORNELLES DA COSTA

Rua Belém, 112 - Centro

95875000 Fazenda Vilanova, RS, Brasil

E-mail: jluana@univates.br 


\section{INTRODUÇÃO}

De acordo com a Organização Mundial da Saúde 1 , a obesidade é uma doença crônica e multifatorial definida pelo acúmulo excessivo de tecido adiposo em um nível que compromete a saúde dos indivíduos. No Brasil, um levantamento com 188 mil brasileiros de todas as idades apontou um aumento da obesidade, constatando que 50\% dos homens e $48 \%$ das mulheres estão com excesso de peso, sendo que $12,4 \%$ dos homens e $16,9 \%$ das mulheres são considerados obesos ${ }^{2}$. Associado a estes dados, sabe-se ainda que $62,1 \%$ da população brasileira é considerada sedentária, sendo este valor maior entre as mulheres, sujeitos com menor poder aquisitivo e entre pessoas com mais idade ${ }^{3}$.

Sabe-se que a obesidade está relacionada a diversos prejuízos na vida dos indivíduos ${ }^{4}$, interferindo também nas questões relacionadas ao aspecto funcional. A capacidade funcional está associada à capacidade para cumprir com eficiência as tarefas do dia a dia $^{5}$. Um estudo avaliou a capacidade funcional de mulheres divididas em três grupos conforme sua classificação no índice de massa corporal (eutrófico, sobrepeso e obeso), sendo observado que as mulheres obesas apresentaram pior capacidade funcional em relação às demais ${ }^{6}$.

Neste sentido, tem sido reportado que reduções mínimas da massa corporal (de 2 a 3\%) em indivíduos com sobrepeso/ obesidade já são suficientes para gerar benefícios à saúde . Assim, a intervenção multidisciplinar, como programas de reeducação alimentar e prática regular de exercícios físicos, tem-se mostrado efetiva para a redução da massa corporal ${ }^{8}$. Em estudo ${ }^{9}$ que acompanhou 5145 americanos (58,7 $\pm 6,8$ anos; $60 \%$ mulheres) participantes de uma intervenção multidisciplinar por um período de um ano, indicou que a magnitude de redução da massa corporal $(-4,77 \pm 7,57 \mathrm{~kg})$ esteve fortemente associada com melhoras no perfil lipídico. Os autores concluíram que até mesmo reduções modestas (5 a 10\%) já estão associadas com melhoras nos fatores de risco para doenças cardiovasculares.

Desta forma, parecem claros os benefícios da redução da massa corporal advindos da prática regular de exercícios físicos e da adoção de hábitos alimentares adequados ${ }^{7,9}$. Além disso, a literatura tem evidenciado que sujeitos obesos apresentam uma pior capacidade funcional ${ }^{6}$. Contudo, são escassas na literatura informações referentes ao impacto do emagrecimento na capacidade funcional. Nesse sentido, o objetivo deste estudo foi avaliar o efeito de um programa de reeducação alimentar na capacidade funcional de mulheres.

\section{MATERIAIS E MÉTODOS}

Este estudo é caracterizado como quase experimental (sem grupo controle) com base de dados retrospectivos.
Foram coletados dados retrospectivos (banco de dados) de 3493 participantes do Programa de Reeducação Alimentar Peso Leve/CERTEL, durante os períodos de 2011 a 2015, no Vale do Taquari, Rio Grande do Sul, Brasil. Os critérios de inclusão para a participação neste programa foram: (1) sujeitos com boas condições físicas (avaliados por meio de anamnese) e (2) assinatura do termo de consentimento livre e esclarecido. Os critérios de exclusão foram definidos como ser do sexo masculino, menores de idade, uma frequência no programa mínima de 50\% e apresentarem os dados completos das variáveis investigadas (preenchimento do questionário HAQ). Este estudo foi aprovado pelo Comitê de Ética da Universidade do Vale do Taquari/UNIVATES (no 198.487).

\section{Programa de Reeducação Alimentar}

O Programa de Reeducação Alimentar Peso Leve foi promovido pela Cooperativa Certel, e constituído por uma equipe multidisciplinar de saúde. O mesmo foi ofertado para a população em geral dos municípios participantes, onde a maior demanda foi composta por sujeitos com sobrepeso ou obesidade. O programa forneceu orientações para uma alimentação balanceada e prática regular de exercícios físicos, tendo como objetivo final o emagrecimento e a melhora da qualidade de vida. Cada grupo (de até 70 integrantes) participou de 20 sessões a cada semestre (aproximadamente duas horas por sessão). A cada sessão, os participantes recebiam orientações sobre aspectos nutricionais e prática de exercícios com um profissional de nutrição e de educação física, respectivamente. Outros profissionais da área da saúde (psicólogo, fisioterapeuta e terapeuta ocupacional) atuaram com foco específico na assistência e qualidade de vida dos participantes em momentos únicos.

As orientações nutricionais ocorriam por meio de palestras expositivas e de algumas dinâmicas de grupo, além de proporcionar um espaço para a discussão e exteriorização dos sentimentos e dificuldades enfrentados no processo de reeducação alimentar. Todos os participantes recebiam um guia o qual serviu como material didático para auxiliar na escolha de ingestão equilibrada de alimentos. A base de informações utilizadas no programa foi a sugerida pelo Guia de Alimentação da População Brasileira indicado pelo Ministério da Saúde. O objetivo principal do nutricionista foi de orientar os sujeitos a alcançar o seu peso desejado, e a partir daí, proporcionar a orientação necessária para mantê-lo ou controlá-lo. As orientações para que o processo de emagrecimento ocorresse eram indicadas para que acontecessem de forma gradual e segura, indicando hábitos alimentares e comportamentais saudáveis e que pudessem ser incorporados de maneira definitiva. Nos encontros eram 
abordado temas diversificados, como: comportamentos e atitudes alimentares; classificação dos nutrientes de acordo com suas funções (plástica - proteínas, energética - carboidratos e gorduras, e reguladora - vitaminas e sais minerais); quantidade de alimentos consumidos a cada refeição, bem como a seleção de alimentos para compor os cardápios variados; equilíbrio alimentar baseados na pirâmide alimentar; a importância de hábitos alimentares (regularidade, mastigação, duração das refeições); bem como, conteúdos diversos relacionados à nutrição e emagrecimento (alimentos diet ou light, relação dos alimentos com prática de exercícios, associação da alimentação com doenças, outros).

Quanto as orientações dos exercícios físicos, tanto a fundamentação teórica como as sessões de atividades de práticas corporais eram ministradas por profissional de Educação Física. Essas atividades visavam o desenvolvimento da flexibilidade articular, força muscular e capacidade cardiorrespiratória e eram realizadas em grupos fazendo uso de exercícios localizados, dança, ginástica coreografada e caminhadas. A prática da caminhada era realizada nas ruas próximas aos locais em que o programa ocorria. Para a realização das demais atividades, usava-se colchonetes, bandas elásticas e exercícios com o próprio peso corporal para sua realização.

\section{Procedimentos de Avaliação}

Quanto as avaliações, parte foram realizadas somente no início e no final do programa, como a estatura, o perímetro abdominal e a capacidade funcional, enquanto a massa corporal e a prática de exercícios físicos foram avaliados semanalmente. Para a avaliação antropométrica foram avaliadas as variáveis massa corporal $(\mathrm{kg})$, estatura $(\mathrm{m})$ e perímetro abdominal $(\mathrm{cm})$, bem como, o cálculo do índice de massa corporal (IMC) $\left(\mathrm{kg} / \mathrm{m}^{2}\right)$. A massa corporal foi mensurada por meio de balança digital (marca Líder; resolução $0,1 \mathrm{~kg}$ ), a estatura por um estadiômetro (Marca WCS, Wood Compact; resolução $0,005 \mathrm{~m})$, o perímetro abdominal $(\mathrm{cm})$ por meio de uma fita métrica metálica (marca Cescorf; resolução $0,1 \mathrm{~cm}$ ), sendo a medida realizada na linha umbilical das participantes. O IMC foi obtido por meio da divisão da massa corporal pelo quadrado da estatura, sendo a participante classificada conforme os critérios sugeridos pela Organização Mundial de Saúde ${ }^{11}$.

Para a mensuração da quantidade de exercícios físicos realizados semanalmente, o primeiro passo foi de orientação dos sujeitos quanto à diferenciação entre atividades físicas e exercícios físicos. Para este estudo, foram consideradas apenas atividades que representassem exercícios físicos, caracterizados como uma sequência sistemática de movimentos de diferentes segmentos corporais, executados de forma planejada e com um determinado objetivo a atingir, como, por exemplo, caminhada, musculação, natação, entre outros. Assim, semanalmente os participantes foram questionados pelo profissional de educação física com a seguinte questão: "qual a quantidade de dias e tempo total de exercícios físicos realizados na semana que se passou (sete dias anteriores)", sendo a resposta registrada em um sistema computadorizado. É importante ressaltar a limitação do presente instrumento. Contudo, considerando que o objetivo principal de tal informação foi a de estratificar os sujeitos em "mais ativos" e "menos ativos", e não avaliar o impacto do volume de exercícios realizados, acredita-se que o mesmo possa servir ao propósito.

Para a avaliação da capacidade funcional, foi utilizado o instrumento Health Assessment Questionnaire (HAQ 20) o qual foi traduzido e adaptado para a língua portuguesa ${ }^{10}$. Este instrumento avalia a dificuldade em realizar as atividades diárias, sendo composto por vinte perguntas em formato de escala Likert, distribuídas em oito domínios: 1: vestir-se; 2: levantar-se; 3: alimentar-se; 4: caminhar; 5: higiene pessoal; 6: alcançar objetos; 7: apreender objetos e 8: outras atividades. Para cada uma das vinte perguntas foi atribuído um valor de 0 a 3 , onde um menor índice representava uma melhor capacidade funcional $(0=$ sem qualquer dificuldade; $1=$ com alguma dificuldade; $2=$ com muita dificuldade; 3 =incapaz de fazer). Para quantificar a capacidade funcional, os índices mais altos de cada domínio foram somados e divididos por oito, obtendo um valor final que variava de 0 a 3 .

\section{Análise Estatística}

As análises foram realizadas utilizando a amostra dividida em subgrupos, sendo estes estratificados pelo IMC, pela magnitude de redução da massa corporal, pela prática de exercícios físicos e a pela idade dos sujeitos. O ponto de corte dos subgrupos de IMC e exercício físico foram definidos por meio dos valores normativos já estabelecidos na literatura, e os subgrupos redução de massa corporal e idade foram definidos por meio do valor da respectiva mediana. Assim, a variável IMC foi estratificada em subgrupo de IMC $\leq 29,9 \mathrm{~kg} /$ $\mathrm{m}^{2}$ e outro de $I M C \geq 30,0 \mathrm{~kg} / \mathrm{m}^{2}$. A redução de massa corporal foi estratificada em um grupo de menor redução $(\leq-4,35 \mathrm{~kg})$ e outro de maior redução (>-4,35 kg). Quanto à prática de exercício físico, os subgrupos foram divididos de acordo com o tempo total de exercício físico praticado semanalmente, formando os subgrupos dos menos ativos ( $<150$ minutos) e dos mais ativos ( $\geq 150$ minutos). Por último, a idade foi estratificada em um subgrupo de menor idade ( $\leq 50$ anos) e um de maior idade (>50 anos). 
Os dados descritivos das variáveis são apresentados por meio de média e desvio padrão. A normalidade dos dados foi testada pelo teste de Kolmogorov-Smirnov, sendo que todas as variáveis apresentaram um comportamento não paramétrico. A comparação das variáveis entre os momentos pré e pós intervenção foi realizada por meio do teste de Wilcoxon para amostras relacionadas, enquanto que a comparação entre um mesmo momento em diferentes subgrupos foi realizada por meio do teste $U$ de Mann-Whitney para amostras independentes. Todos os procedimentos foram realizados no programa SPSS versão 20.0, sendo adotado um nível de significância de $p \leq 0,05$.

\section{RESULTADOS}

Dentre os dados dos 3493 participantes do programa, os critérios de exclusão eliminaram 2699 sujeitos do estudo, sendo 466 indivíduos por serem do sexo masculino, sete menores de idade, 760 por não atingirem o mínimo de frequência no programa e 1466 por não apresentarem os dados completos, e desta forma, a amostra foi composta por 794 mulheres. O perfil destas 794 mulheres apresentou uma média de idade de 48,39 $\pm 12,32$ anos, estatura de $1,61 \pm 0,06 \mathrm{~m}$, massa corporal de $80,66 \pm 14,58 \mathrm{~kg}$ e IMC de $30,79 \pm 5,21 \mathrm{~kg} / \mathrm{m}^{2}$.

A Tabela 1 apresenta os valores de média e desvio padrão pré e pós intervenção das variáveis massa corporal, IMC, perímetro abdominal e capacidade funcional dos sujeitos analisados em um único grupo. Os resultados indicaram que todas as variáveis apresentaram reduções significativas $(p<0,001)$ quando comparados os valores do início para o final do programa. Quando avaliada a capacidade funcional pré e pós intervenção nos diferentes subgrupos, observou-se uma redução significativa $(p<0,001)$ dos valores em todas as análises realizadas (Tabela 2). Além disso, quando comparada a capacidade funcional em um mesmo momento, tanto na fase pré como pós intervenção, os sujeitos mais pesados (subgrupo de IMC obeso) e mais velhos, apresentaram valores superiores aos seus pares mais leves e mais novos

Tabela 1. Média e desvio padrão pré e pós intervenção em grupo único das variáveis massa corporal, IMC, perímetro abdominal e capacidade funcional (HAQ 20).

\begin{tabular}{lcccc}
\hline Variáveis & n & \multicolumn{2}{c}{ Média \pm DP } & Pré \\
Massa corporal $(\mathrm{kg})$ & 794 & $80,66 \pm 14,58$ & $75,48 \pm 14,03$ & $<0,001$ \\
IMC $\left(\mathrm{kg} / \mathrm{m}^{2}\right)$ & 794 & $30,79 \pm 5,21$ & $28,82 \pm 5,03$ & $<0,001$ \\
Perímetro abdominal $(\mathrm{cm})$ & 498 & $98,58 \pm 11,57$ & $91,19 \pm 11,27$ & $<0,001$ \\
HAQ 20 (u.a.) & 794 & $0,17 \pm 0,31$ & $0,07 \pm 0,21$ & $<0,001$ \\
\hline
\end{tabular}

u.a.: unidade arbitrária; IMC: índice de massa corporal. $(p<0,001)$, ou seja, sujeitos mais pesados e mais velhos iniciaram e finalizaram com uma pior capacidade funcional (Tabela 2).

Tabela 2. Média e desvio padrão da variável capacidade funcional (HAQ20) nos subgrupos quanto ao IMC, redução de massa corporal, prática de exercícios físicos e idade.

\begin{tabular}{|c|c|c|c|c|}
\hline \multirow{2}{*}{ Subgrupos } & \multirow{2}{*}{$\mathbf{n}$} & \multicolumn{2}{|c|}{ HAQ (u.a.) } & \multirow{2}{*}{$p$} \\
\hline & & Pré & Pós & \\
\hline \multicolumn{5}{|l|}{ IMC (kg/m²) } \\
\hline Até 29,9 & 396 & $0,08 \pm 0,18^{*}$ & $0,03 \pm 0,11^{*}$ & $<0,001$ \\
\hline Maior que 29,9 & 398 & $0,24 \pm 0,37$ & $0,10 \pm 0,26$ & $<0,001$ \\
\hline \multicolumn{5}{|l|}{$\begin{array}{l}\text { Redução de massa } \\
\text { corporal }(\mathrm{kg})\end{array}$} \\
\hline Até -4,35 & 397 & $0,15 \pm 0,29$ & $0,06 \pm 0,19$ & $<0,001$ \\
\hline Maior que $-4,35$ & 397 & $0,18 \pm 0,32$ & $0,07 \pm 0,22$ & $<0,001$ \\
\hline \multicolumn{5}{|l|}{$\begin{array}{l}\text { Exercício físico } \\
\text { (min/semanal) }\end{array}$} \\
\hline$\leq 150$ & 377 & $0,19 \pm 0,34$ & $0,08 \pm 0,22$ & $<0,001$ \\
\hline$\geq 150$ & 417 & $0,14 \pm 0,27$ & $0,05 \pm 0,18$ & $<0,001$ \\
\hline \multicolumn{5}{|l|}{ Idade (anos) } \\
\hline $18-50$ & 413 & $0,11 \pm 0,22^{*}$ & $0,04 \pm 0,12^{*}$ & $<0,001$ \\
\hline $51-80$ & 381 & $0,22 \pm 0,36$ & $0,10 \pm 0,26$ & $<0,001$ \\
\hline
\end{tabular}

u.a.: unidade arbitrária; IMC: índice de massa corporal; valor de p: nível de significância entre os momentos pré e pós para a variável capacidade funcional no respectivo subgrupo $(p \leq 0,001)$

* Indica diferenças significativas entre os subgrupos em um mesmo momento $(p \leq 0,001)$.

\section{DISCUSSÃO}

O resultado principal do presente estudo indicou uma redução significativa $(p<0,001)$ da massa corporal $(\approx 6,4 \%)$, do IMC e do perímetro abdominal, bem como, uma melhora significativa $(p<0,001)$ da capacidade funcional. Em estudo ${ }^{8}$ no qual foi realizada uma intervenção em um SPA de Santa Catarina (Brasil), com acompanhamento nutricional e a prática de exercícios físicos, os resultados indicaram que os sujeitos ( $n=60 ; 60 \%$ mulheres; entre 45 e 60 anos, IMC $>30 \mathrm{~kg} / \mathrm{m}^{2}$ ) que foram submetidos ao programa apresentaram uma redução da massa corporal de aproximadamente $3 \mathrm{~kg}$. O valor de redução da massa corporal foi inferior à média do presente estudo, possivelmente pelo fato de ser um estudo com um menor tempo de intervenção. Sabe-se que reduções de 5 a $10 \%$ da massa corporal promovem uma melhora significativa no controle metabólico e nos níveis de pressão arterial, além de reduzir o índice de mortalidade relacionada com as dislipidemias ${ }^{12}$. Além disso, eliminar cerca de $10 \mathrm{~kg}$ da massa corporal reduz cerca de 20 a $25 \%$ o risco de mortalidade decorrente de complicações associadas à obesidade ${ }^{13}$. Ainda, embora a redução dos valores de IMC tenham sido modestas $\left(\approx 2 \mathrm{~kg} / \mathrm{m}^{2}\right)$ e que este valor possivelmente represente uma diferença clinicamente pouco 
expressiva, salienta-se que considerando a média de estatura do presente grupo, cada unidade de IMC alterada representa em torno de 2,6 kg de massa corporal.

No presente estudo foi constatado que após uma intervenção de 20 semanas, uma melhora significativa da capacidade funcional foi encontrada entre os participantes. Outros autores ${ }^{14}$ identificaram uma mediana da capacidade funcional de 1,13 pelo HAQ20 em sujeitos obesos (IMC entre 36 e $52 \mathrm{~kg} / \mathrm{m}^{2}$ ), sendo esta capacidade considerada moderada. Em contrapartida, outro estudo ${ }^{15}$ comparando a capacidade funcional de mulheres com hipertrofia mamária (sobrepeso; $\mathrm{n}=50$ ) com mulheres com mamas normais (eutróficas; $\mathrm{n}=50$ ), identificaram uma pior capacidade funcional no primeiro grupo (HAQ20: 0,71 $\pm 0,52$ vs $0,10 \pm 0,21$ ). No presente estudo, embora os sujeitos apresentassem um IMC médio consideravelmente elevado $\left(\mathrm{IMC} \approx 31 \mathrm{~kg} / \mathrm{m}^{2}\right)$, os dados iniciais da capacidade funcional não podem ser considerados incapacitantes (HAQ pré=0,17). Além disso, considerando apenas os sujeitos com um score médio geral superior a "um", na fase inicial da intervenção, somente $14 \%$ dos participantes estavam classificados com incapacidade, diminuindo esta quantidade para $6 \%$ na fase final do programa.

Com relação aos resultados estratificados em subgrupos conforme o IMC constatou-se que ambos os grupos melhoraram sua capacidade funcional após a intervenção, sendo que o grupo de IMC maior apresentou valores da capacidade funcional três vezes piores que o grupo de IMC menor, tanto na fase pré como pós intervenção. Isto indica que possuir um IMC elevado pode ser um dos fatores determinantes para uma piora na capacidade funcional. De acordo com a literatura ${ }^{6,16}$, parece evidente que, conforme o sujeito aumenta seus valores de IMC, maior o comprometimento da sua capacidade funcional, gerando assim um aumento na carga de trabalho na realização de suas tarefas ${ }^{17}$. Além disso, não somente a capacidade funcional é comprometida por conta do peso excessivo, como também a capacidade cardiorrespiratória ${ }^{18}$ e o desempenho físico ${ }^{19}$, pois a demanda metabólica é aumentada pelo trabalho muscular adicional necessário para compensar o excesso da massa corporal ${ }^{20-21}$.

Referente à magnitude de redução da massa corporal, tanto o grupo de menor redução quanto o grupo de maior redução apresentaram melhoras significativas na capacidade funcional após a intervenção, não apresentando diferenças significativas entre os subgrupos em nenhum momento. Além disso, o grupo de maior redução de massa corporal também apresentou um IMC inicial mais elevado, bem como, realizou uma maior quantidade de exercícios físicos (dias e tempo total semanal) durante o programa. Villareal et al. ${ }^{22}$ realizaram uma intervenção com duração de um ano em 93 idosos, visando comparar os resultados de uma bateria de testes físicos e a capacidade funcional (por meio de questionário), entre quatro grupos experimentais: (1) grupo controle, (2) grupo que realizou apenas exercícios físicos, (3) grupo que realizou apenas dieta e (4) grupo que realizou dieta e exercício. Ao final do estudo foi verificado que o desempenho físico melhorou 12, 15 e $21 \%$, enquanto que na capacidade funcional houve um incremento de 4, 6 e 10\%, nos grupos dieta, exercício e dieta-exercício, respectivamente, sem alterações no grupo controle. É importante salientar que somente os grupos dieta e dieta-exercício apresentaram reduções significativas na massa corporal (10 e $9 \%$, respectivamente), resultado este não observado no grupo exercício e controle. Assim, constata-se que o grupo exercício obteve incremento similar na capacidade física e funcional, sem uma concomitante redução da massa corporal, sugerindo que esta magnitude possa estar relacionada a outros fatores, corroborando com os dados encontrados por este estudo. Dessa forma, pode-se inferir que não é necessário apresentar uma grande redução na massa corporal para se obter uma melhora na capacidade funcional, indicando que uma redução de aproximadamente $2 \mathrm{~kg}$ na massa corporal já pode produzir um efeito positivo na capacidade funcional.

Em relação à comparação dos subgrupos relacionados à prática de exercício físico, constatou-se que tanto o grupo de maior volume de exercícios físicos quanto o de menor, obtiveram melhoras significativas na sua capacidade funcional após a intervenção. Estas semelhanças ocorreram mesmo com o grupo de maior volume apresentando aproximadamente valores três vezes superiores no tempo total de prática semanal, bem como, apresentando o dobro da frequência semanal de exercícios. Vários estudos têm indicado que diferentes formas de exercícios físicos podem contribuir na melhora da capacidade funcional e consequentemente na qualidade de vida, sendo sugerida a força máxima como um dos componentes determinantes ${ }^{23,24}$.

Embora pareça evidente que a prática de exercícios físicos, e consequente melhora da aptidão física, contribuam numa melhora da capacidade funcional, é importante observar que tal aspecto torna-se fundamental quando nos referimos à inclusão de exercícios na rotina de sujeitos previamente sedentários ou com algum tipo de comprometimento físico. Este aspecto tem sido evidenciado em estudo ${ }^{25}$ no qual não se encontrou relação entre a capacidade funcional e os níveis de força em mulheres idosas, e sim justificado pelo nível elevado de atividade física habitual da amostra. Assim, uma possível explicação seria a existência de um limiar funcional, ou seja, os benefícios do exercício físico na capacidade funcional só ocorreriam em pessoas mais debilitadas (àquelas 
classificadas abaixo deste limiar ${ }^{26}$. Desta forma, conforme os dados encontrados no presente estudo, sugere-se que o volume de exercícios físicos não seja determinante na melhora funcional, sendo que pequenas quantidades (dois dias e 100 minutos semanais) já seriam suficientes para o incremento da capacidade funcional.

Quando comparada a capacidade funcional em grupos distintos quanto à idade, o presente estudo constatou que ambos os grupos apresentaram melhoras significativas na capacidade funcional em similar magnitude. Contudo, tanto na fase pré como pós intervenção, o grupo mais novo $(38,7 \pm 7,5$; entre 18 e 50 anos) apresentou aproximadamente a metade do comprometimento da capacidade funcional quando comparado ao grupo mais velho $(58,9 \pm 6,5$; entre 50 e 80 anos). Ferreira et al. ${ }^{27}$ compararam a capacidade funcional de mulheres jovens $(23,2 \pm 2,8$ anos) e idosas $(62,5 \pm 8,2$ anos) por meio da bateria de testes da AAHPERD, indicando um desempenho superior entre as mulheres mais jovens. Araújo et al. ${ }^{28}$ também constataram que a idade está fortemente associada à perda de capacidade funcional, ou seja, quanto maior a idade do sujeito, maior será a probabilidade de apresentar níveis de incapacidade relacionados com as atividades de vida diária. Neste sentido, tem sido evidenciado que conforme os indivíduos vão envelhecendo as funções fisiológicas vão reduzindo ${ }^{27}$. A diminuição da massa muscular e a menor ativação das unidades motoras são fatores que levam a um declínio de 10 e $15 \%$ por década no nível de força do sujeito a partir dos 50 anos $^{29}$. Desta forma, ocorre uma redução da função motora e da integridade do sistema neuromuscular que consequentemente compromete a capacidade funcional ${ }^{30}$.

Como limitações deste estudo, consideramos os seguintes aspectos: (1) a avaliação da capacidade funcional ter sido realizada por meio de questionário, (2) o registro da prática de exercícios ser determinada por auto-relato em instrumento não validado e (3) a falta de um grupo controle, o qual pode gerar alguns vieses de interpretação científica. Contudo, salientamos a validade externa do estudo, visto que o mesmo ocorreu dentro de um programa realizado dentro da comunidade. Além disso, acredita-se que uma vez obedecidos os protocolos sugeridos e a padronização de coleta das informações, tais limitações possam ser minimizadas.

Assim, conclui-se que a participação de mulheres em um programa de reeducação alimentar promove uma melhora da capacidade funcional, bem como reduz vários parâmetros da composição corporal. Além disso, a quantidade de exercícios físicos realizados, assim como a magnitude de redução da massa corporal, não são fatores determinantes da capacidade funcional, ao contrário da idade e do IMC, de maneira que sujeitos mais velhos e com maior IMC apresentam um maior comprometimento da capacidade funcional. Por fim, é importante mencionar que, como este não é um estudo controlado, estes resultados possam estar associados não somente a redução da massa corporal, como também a outros aspectos, como a prática de exercícios físicos, aspectos psicológicos, entre outros.

\section{REFERÊNCIAS}

1. World Health Organization. Draft who global action on physical activity 2018-2030 [Internet]. WHO; 2017 [citado 23 de outubro de 2017]. Disponível em: http://www.who.int/ncds/governance/ physical_activity_plan/en/

2. Brasil. Instituto Brasileiro de Geografia e Estatística. Pesquisa de orçamentos familiares 2008-2009: antropometria e estado nutricional de crianças, adolescentes e adultos no Brasil [Internet]. Rio de Janeiro: IBGE; 2010 [citado 24 de novembro de 2016]. Disponível em: http://www.abeso.org.br/uploads/ downloads/71/553a23f27da68.pdf

3. Brasil. Instituto Brasileiro de Geografia e Estatística. Práticas de esporte e atividade física: 2015 [Internet]. Rio de Janeiro: IBGE; 2017 [citado 23 de outubro de 2017]. Disponível em: https:// biblioteca.ibge.gov.br/visualizacao/livros/liv100364.pdf

4. Peixoto MRG, Benício MHA, Latorre MRDO, Jardim PCBV. Circunferência da cintura e índice de massa corporal como preditores da hipertensão arterial. Arq Bras Cardiol. 2006;87(4): 462-70. https://doi.org/10.1590/S0066-782X2006001700011

5. Grigoletto ME, Brito CJ, Heredia JR. Treinamento funcional: funcional para que e para quem? Rev Bras Cineantropom Desempenho Hum. 2014;16(6):714-19. https://doi.org/10.5007/1980-0037. 2014v16n6p714

6. Orsi JVA, Nahas FX, Gomes HC, Andrade CHV, Veiga DF, Novo NF, Ferreira LM. Impacto da Obesidade na Capacidade Funcional de Mulheres. Rev Assoc Med Bras. 2008;54(2):106-09. https://doi. org/10.1590/S0104-42302008000200010

7. Donnelly JE, Jacobsen DJ, Kirk E, Dubose K, Hyder M, Bailey B, Washburn $R$. The role of exercise for weight loss and maintenance. Best Pract Res Clin Gastroenterol. 2004;18(6):1009-29. https://doi. org/10.1016/S1521-6918(04)00083-6

8. Schneider C, Veras A, Nascimento MB, Liberali R. Efeitos de um programa de intervenção nutricional sobre a composição corporal e os hábitos alimentares de obesos em spa / SC. Rev Bras Obes Nut Emagrec. 2007;1(1):90-101.

9. Wing RR, Lang W, Wadden TA, Safford M, Knowler WC, Bertoni AG, Hill JO, Brancati FL, Peters A, Wagenknecht L. Benefits of modest weight loss in improving cardiovascular risk factors in overweight and obese individuals with type 2 diabetes. Diabetes Care. 2011;34(7):1481-86. https://doi.org/10.2337/dc10-2415

10. Ferraz MB. Tradução para português e validação do questionário para avaliar a capacidade funcional "Stanford Health Assessment Questionnaire" [tese de doutorado]. São Paulo: Universidade Federal de São Paulo; 1990.

11. Organização Mundial de Saúde - OMS. Obesity: preventing and managing the global epidemic [Internet]. WHO; 2000 [citado 26 de outubro de 2017]. Disponível em: http://www.who.int/nutrition/ publications/obesity/WHO_TRS_894/en/ 
12. Negrão CE, Barreto ACP. Cardiologia do exercício: do atleta ao cardiopata. São Paulo: Manole; 2005.

13. Jung RT. Obesity as a disease. Br Med Bull. 1997;57(2):307-21. https://doi.org/10.1093/oxfordjournals.bmb.a011615

14. Soares KKD, Gomes ELFD, Junior AB, Oliveira LVF, Sampaio LMM, Costa D. Avaliação do Desempenho Físico e Funcional Respiratório em Portadores de Obesidade. Fisioter Mov. 2011;24(4):697-04. https://doi.org/10.1590/S0103-51502011000400014

15. Araújo CD, Gomes HC Veiga DF, Hochman B, Fernandes PM, Novo NF, Ferreira LM. Influência da Hipertrofia Mamária na Capacidade Funcional das Mulheres. Rev Bras Reumatol. 2007;47(2):91-96. https://doi.org/10.1590/S0482-50042007000200003

16. Oeser A, Chung CP, Asanuma Y, Avalos I, Stein M. Obesity is an independent contributor no functional capacity and inflammation in systemic lupus erythematosus. Arthritis Rheum. 2005;52(11): 3651-9. https://doi.org/10.1002/art.21400

17. Hulens M, Vansant G, Claessens AL, Lysens R, Muls E. Predictors of 6-minute walk test results in lean, obese and morbidly obese women. Scand J Med Sci Sports. 2003;13(2):98-105. https://doi. org/10.1034/j.1600-0838.2003.10273.x

18. Eisenmann JC, Wickel EE, Welk GJ, Blair SN. Relationship between adolescent fitness and fatness and cardiovascular disease risk factors in adulthood: the Aerobics Center Longitudinal Study (ACLS). Am Heart J. 2005;149(1):46-53. https://doi.org/10.1016/j. ahj.2004.07.016

19. Gontijo PL, Lima TP, Costa TR, Reis EP, Cardoso FPF. Correlação da espirometria com o teste de caminhada de seis minutos em eutróficos e obesos. Rev Assoc Med Bras. 2011;57(4):387-93. https://doi.org/10.1590/S0104-42302011000400010

20. Gallagher MJ, Franklin BA, Ehrman JK, Keteyian SJ, Brawner CA, Jong AT, McCullough PA. Comparative impact of morbid obesity vs heart failure on cardiorespiratory fitness. Chest. 2005;127(6):2197-03. https://doi.org/10.1378/chest.127.6.2197

21. World Health Organization. Global strategy on diet, physical activity and health [Internet]. WHO; 2004 [citado 2017 nov 03]. [online]. Disponível em: http://www.who.int/dietphysicalactivity/strategy/ eb11344/strategy_english_web.pdf
22. Vilarreal DT, Chode S, Parimi M, Sinacore DR, Shah K, Hilton T, Armamento-Villareal R, Napoli N, Qualls C, Shah K. Weight loss, exercise, or both and physical function in obese older adults. $\mathrm{N}$ Engl J Med. 2011;364(13):1218-29. https://doi.org/10.1056/ NEJMoa1008234

23. Tiggemann CL, Dias CP, Radaelli R, Massa JC, Bortoluzzi R, Schoeneel MCW, Noll M, Alberton CL, Kruel LF. Effect of traditional resistance and power training using rated perceived exertion for enhancement of muscle strength, power, and functional performance. Age (Dordr). 2016;38(2):42. https://doi.org/10.1007/s11357-016-9904-3

24. Schuch FB, Schoenell MCW, Tiggemann CL, Noll M, Alberton CL, Kruel LFM. The effects of water-based strength exercise on quality of life in young women. Sport Sci Health. 2016;12:105-12. https:// doi.org/10.1007/s11332-016-0261-5

25. Roncato M, Galarza E, Freire B, Tiggemann CL, Dias CP. Correlação da força e composição corporal com a capacidade funcional em mulheres idosas. Rev Bras Cienc Mov. 2014;22(1):122-30. https:// doi.org/10.18511/0103-1716/rbcm.v22n1p122-130

26. Ferrucci L, Guralnik JM, Buchner D, Kasper J, Lamb SE, Simonsick EM, Corti MC, Bandeen-Roche K, Fried LP. Departures from linearity in the relationship between measures of muscular strength and physical performance of the lower extremities: the Women's Health and Aging Study. J Gerontol A Biol Sci Med Sci. 1997;52(5): M275-85. https://doi.org/10.1093/gerona/52A.5.M275

27. Ferreira L, Barbosa TD, Gobbi S, Arantes LM. Capacidade funcional em mulheres jovens e idosas: projeções para uma adequada prescrição de exercícios físicos. Rev Educ Fís. 2008;19(3):403-12 https://doi.org/10.4025/reveducfis.v19i3.5995

28. Araújo F, Ribeiro JLB, Oliveira A, Pinto C. Validação do Índice de Barthel numa amostra de idosos não institucionalizados. Rev Port Saúde Pública. 2007;25(2):59-66.

29. Deschenes MR. Effects of aging on muscle fiber type and size. Sports Med. 2004;34(12):809-24. https://doi.org/10.2165/00007256200434120-00002

30. Greenlund LJS, NAIR KS. Sarcopenia: consequences, mechanisms, and potential therapies. Mech Ageing Dev. 2003;124(3):287-99. https://doi.org/10.1016/S0047-6374(02)00196-3 